\title{
A randomised controlled trial of nasal decongestant to treat obstructive sleep apnoea in people with cervical spinal cord injury
}

\author{
Nirupama S. Wijesuriya ${ }^{1} \cdot$ Danny J. Eckert ${ }^{1,2} \cdot$ Amy S. Jordan $^{3,4} \cdot$ Rachel Schembri $^{3} \cdot$ Chaminda Lewis $^{1}$. \\ Hailey Meaklim ${ }^{3}$ - Lauren Booker ${ }^{3}$. Doug Brown ${ }^{5} \cdot$ Marnie Graco $\mathbb{D}^{3,4} \cdot$ David J. Berlowitz $\mathbb{D}^{3,4,6}$
}

Received: 30 September 2018 / Revised: 27 January 2019 / Accepted: 30 January 2019 / Published online: 13 February 2019

(C) International Spinal Cord Society 2019

\begin{abstract}
Study design Prospective, double-blind, randomised, placebo-controlled, cross-over trial of nasal decongestion in tetraplegia.

Objectives Tetraplegia is complicated by severe, predominantly obstructive, sleep apnoea. First-line therapy for obstructive sleep apnoea is nasal continuous positive airway pressure, but this is poorly tolerated. High nasal resistance associated with unopposed parasympathetic activation of the upper airway contributes to poor adherence. This preliminary study tested whether reducing nasal decongestion improved sleep.

Setting Participants' homes in Melbourne and Sydney, Australia.

Methods Two sleep studies were performed in participants' homes separated by 1 week. Participants were given a nasal spray $(0.5 \mathrm{~mL}$ of $5 \%$ phenylephrine or placebo) in random order and posterior nasal resistance measured immediately. Outcomes included sleep apnoea severity, perceived nasal congestion, sleep quality and oxygenation during sleep.

Results Twelve middle-aged (average (SD) 52 (12) years) overweight (body mass index $25.3(6.7) \mathrm{kg} / \mathrm{m}^{2}$ ) men (C4-6, AIS A and B) participated. Nasal resistance was reduced following administration of phenylephrine $(p=0.02$; mean between treatment group difference $-5.20: 95 \%$ confidence interval $\left.-9.09,-1.32 \mathrm{cmH}_{2} \mathrm{O} / \mathrm{L} / \mathrm{s}\right)$. No differences were observed in the apnoea hypopnoea index ( $p=0.15 ;-6.37:-33.3,20.6$ events $/ \mathrm{h})$, total sleep time $(p=0.49 ;-1.33:-51.8,49.1 \mathrm{~min})$, REM sleep $\%(p=0.50 ; 2.37:-5.6,10.3)$, arousal index $(p=0.76 ; 1.15:-17.45,19.75), 4 \%$ oxygen desaturation index $(p=$ $0.88 ; 0.63:-23.5,24.7$ events $/ \mathrm{h}$ ), or the percentage of mouth breathing events $(p=0.4 ;-8.07:-29.2,13.0)$ between treatments. The apnoea hypopnoea index did differ between groups, however, all except one participant had proportionally more hypopnoeas than apnoeas during sleep after decongestion.

Conclusions These preliminary data found that phenylephrine acutely reduced nasal resistance but did not significantly change sleep-disordered breathing severity.
\end{abstract}

David J. Berlowitz

david.berlowitz@austin.org.au

1 Neuroscience Research Australia (NeuRA), Randwick, NSW, Australia

2 University of New South Wales, Sydney, NSW, Australia

3 Institute of Breathing and Sleep (IBAS), Melbourne, VIC, Australia

4 The University of Melbourne, Melbourne, VIC, Australia

5 Spinal Research Institute, Heidelberg, VIC, Australia

6 Department of Respiratory and Sleep Medicine, Austin Health, Melbourne, VIC, Australia

\section{Introduction}

Spinal cord injury (SCI) is a debilitating condition associated with severe physical disability, reduced quality of life and a high life-time economic cost [1]. Obstructive sleep apnoea (OSA) is a common secondary complication associated with cervical SCI or tetraplegia, which develops within weeks of injury [2]. Tetraplegia prevalence rates of OSA are reported as up to $70 \%[2,3]$, two to seven times more prevalent than the general population [4]. OSA in people with tetraplegia is associated with day-time sleepiness and fatigue [5, 6], impaired cognitive function $[7,8]$ and a decline in quality of life beyond that attributable to the SCI alone [4].

The pathogenesis of OSA results from a combination of anatomical and non-anatomical physiological traits 
including high upper airway collapsibility, low arousal threshold, high loop gain and low muscle responsiveness [9]. While the relative contributions of these factors are likely to be different in tetraplegia [10], anatomical and physiological changes associated with injury, such as altered autonomic [11], neuromuscular [12] and respiratory control [13], increased adipose tissue and neck circumference [2, 14], and increased nasal resistance [15, 16], may increase the risk of OSA in people with tetraplegia.

Increased nasal resistance is a risk factor for OSA. Nasal resistance is increased in patients with SCI and OSA compared with non-disabled people with OSA, and may represent a distinct pathophysiological phenotype in this population $[15,16]$. Injury to the cervical spinal cord disrupts the supraspinal inputs to spinal sympathetic neurons below the level of injury, leaving parasympathetic drive largely unopposed. The autonomic imbalance increases vasodilation and mucosal thickening in the nose, which may reduce nasal patency and increase nasal resistance. Nasal resistance is estimated to account for 30-50\% of total upper airway resistance in non-disabled (non-neurologically impaired) populations and it is estimated that $15 \%$ of people with OSA have increased nasal obstruction [17]. Elevated nasal resistance increases respiratory drive, upper airway collapsibility and the risk of sleep-disordered breathing in the non-disabled [18]. Nasal resistance is higher in ablebodied snorers in the supine position compared with upright [15]. Nasal congestion can also encourage mouth breathing, which is associated with increased upper airway resistance and airway collapsibility [17, 18]. Mouth breathing can further increase upper airway resistance by altering surface tension forces and reducing upper airway dilator muscle efficiency [17]. Increased nasal congestion is associated with lower adherence to continuous positive airway pressure (CPAP) therapy in the non-disabled with OSA [18] and has been previously proposed to contribute to poor adherence to CPAP therapy in tetraplegia [2, 19]. As such, chronically elevated nasal resistance could be a potential therapeutic target in people with tetraplegia and OSA. Our group has previously demonstrated that upper airway resistance is increased in tetraplegia and that topical phenylephrine (PE) can acutely reduce this resistance to the same level as observed in non-disabled controls [16]. We therefore undertook a randomised controlled cross-over trial of nasal decongestion in people with tetraplegia and OSA to determine the effect on OSA severity.

\section{Method}

Participants had chronic tetraplegia (T1 lesion or higher; $>1$-year post injury) with injury completeness classified on the American Spinal Injury Association (ASIA) impairment scale (AIS) as A or B [20], and were aged over 18 years of age. People who had previously participated in related research projects at the Institute for Breathing and Sleep in Melbourne, and Neuroscience Research Australia in Sydney were invited to participate. Participants had a polysomnography (PSG) confirmed diagnosis of OSA within the preceding 12 months, or underwent repeat PSG to confirm previously reported OSA (apnoea hypopnoea index, AHI of $>10$ events/h) [21]. Patients were excluded if they had an $\mathrm{AHI}<10$ events/h, uncontrolled hypertension, history of significant head injury, ongoing hypercapnic ventilatory failure, fixed nasal obstruction (e.g., nasal fracture or deviated septum), were unable to provide informed consent, used bronchodilators, corticosteroid inhalers or other medication that could influence nasal patency, or had an allergy to PE or lignocaine medications.

A within subjects cross-over, double-blind, randomised, proof of concept trial was implemented. Two study visits were carried out in the participants' homes, with a 1-week washout period (Fig. 1). At each visit, PE or placebo was administered (in random sequence), nasal resistance measured, and an overnight PSG carried out. Randomisation of treatment order was prepared by a researcher independent from the study and sequential opaque envelopes used to ensure allocation concealment. Doses were $\sim 0.5 \mathrm{~mL}$ of both an over the counter topical atomised nasal spray (Nyal Decongestant, phenyepherine hydrochloride $5 \mathrm{mg} / \mathrm{mL}$ ) and a normal saline placebo (FESS Sensitive Noses $9 \mathrm{mg} / \mathrm{mL}$ sodium chloride). Bottle labelling were removed and replaced with a simple 'A' or 'B' label. All participants provided informed consent (written, or witnessed verbal) for participation, and the study was registered (Australia New Zealand Clinical Trials Registry ACTRN12615000229572) and approved by the NSW Health South Eastern Sydney Local Health District and Austin Health human research ethics committees.

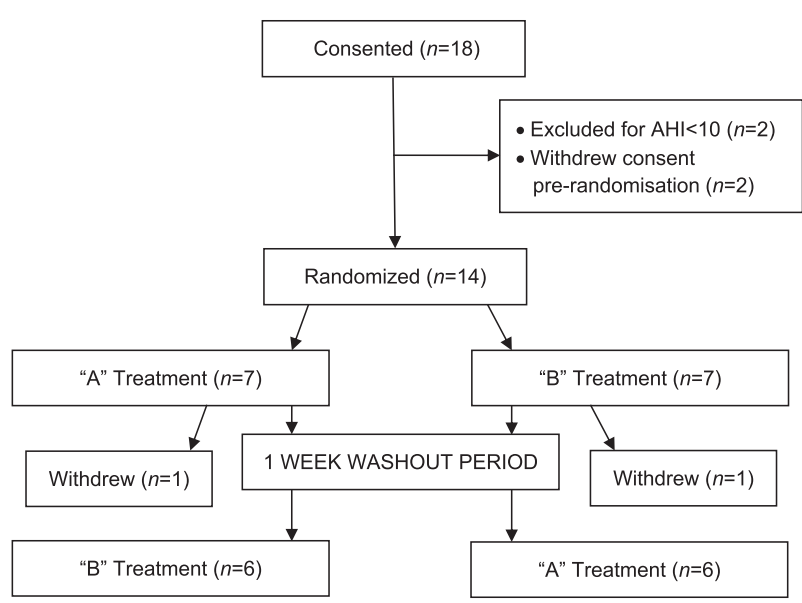

Fig. 1 Study recruitment and participant flowchart 
At each visit, two researchers arrived approximately $1 \mathrm{~h}$ before the participants' usual bedtime. Electrodes and sensors were applied for PSG with a Compumedics Somte (Abbotsford, Australia) ambulatory device, measuring electroencephalography, electrooculography, electromyography, electrocardiography, respiratory effort, nasal flow, oronasal thermistor, limb movement, position and snore. Two questionnaires assessing subjective nasal congestion were administered. A Borg-like scale of Nasal Obstruction asking how blocked the nose feels 'right now' [15] while nasal congestion over 'the past week' was assessed using the Congestion Quantifier five-item test [22], which asks five questions about the frequency of symptoms and outcomes.

Supine, posterior nasal resistance was measured using a pressure transducer tipped catheter (Mikro-tip catheter transducers Model MPC-500; Millar Instruments Inc., Houston, TX) inserted into participants' nose $0.5-1 \mathrm{~cm}$ distal to the nasopharyngeal wall (to measure at the choanae). Participants were fitted with a modified leak proof (sealed exhalation port) nasal mask (ComfortGel Blue nasal mask; Philips Respironics, Pennsylvania, USA). Airflow was measured using a pneumotachograph (Hans Rudolph Inc., 3700, Kansas City, USA) and mask pressure was recorded using a differential pressure transducer attached to the mask and connected to a pneumotachometer (model RSS100-HR, Hans Rudolph Inc., Kansas City, USA). Data were acquired using a 16-bit analogue to digital converter (CED 1401; Cambridge Electronic Design, Cambridge, UK) and Spike 2 software (version 7.2; Cambridge Electronic Design, Cambridge, UK). Participants were instructed to breath comfortably through their nose while baseline nasal resistance was measured for $10 \mathrm{~min}$. Following this, two doses of nasal spray were administered to each nostril and participants were instructed to breathe through their nose for a further $10 \mathrm{~min}$. The average value of the nasal resistance during the $10 \mathrm{~min}$ of measurement was used for analyses. Following nasal resistance measures, instrumentation was removed, Borg nasal obstruction questionnaire repeated and PSG recording commenced.

\section{Data analyses}

PSG data were analysed centrally by one experienced sleep scientist using 2012 American Association of Sleep Medicine criteria [21]. Sleep and respiratory indices were calculated for AHI, total sleep time, route of breathing (proportion of respiratory events identified as being through the oral only route, or oral/nasal), arousal index, $4 \%$ oxygen desaturation index and slow wave and Rapid Eye Movement (REM) sleep percentages. Overnight respiratory and sleep events were quantified both for the full night and separately for the first and second halves of the night. The
AHI and total sleep time in the first half of the night were the primary outcome measures. As previously described, nasal resistance was calculated at an inspiratory flow rate of $0.2 \mathrm{~L} / \mathrm{s}$ (change in pressure/flow) on a breath-by-breath basis, as the pressure difference between mask pressure and choanal pressure [15].

Statistical analyses were conducted blinded to treatment allocation. Analysis of variance for a $2 \times 2$ cross-over study were carried out in Stata 14.2 using 'pkcross' and accounting for randomisation order, drug allocation, visit number, participant and outcome measures. The 'pkcross' command accounts for the period effect of cross-over trials. In addition, the short-acting treatment effect of PE, the stable underlying condition (nasal resistance) and the washout period between conditions, all minimise any potential impact of time trends in this cross-over study. The primary results are presented as the between group, treatment effect; PE versus control comparisons. Paired $t$-test analyses were conducted as sensitivity analyses for primary outcome measures and significant findings.

\section{Results}

Eighteen people were enrolled in the study between and February 2015 and August 2016 (Fig. 1 and Table 1). No significant treatments effects (PE versus control) were observed for AHI, total sleep time, REM sleep time, arousal index, $4 \%$ oxygen desaturation index or route of breathing (in the first half of the night, or the full night) between nights where PE or placebo were administered (Table 2). Nasal resistance was reduced by $72 \%$ following administration of $\mathrm{PE}(p=0.02$; mean difference -5.20 : $95 \%$ confidence interval $\left.-9.09,-1.32 \mathrm{cmH}_{2} \mathrm{O} / \mathrm{L} / \mathrm{s}\right)$. Self-reported

Table 1 Participant demographic and injury information

\begin{tabular}{ll}
\hline & $N(\%)$ or mean $(\mathrm{SD})$ \\
\hline$N$ & 12 \\
Sex & Male $(100 \%)$ \\
Age (years) & $52.1(12.1)$ \\
Body mass index $\left(\mathrm{kg} / \mathrm{m}^{2}\right)$ & $25.3(6.7)$ \\
Injury level & \\
C4 & $3(25 \%)$ \\
C5 & $5(42 \%)$ \\
C6 & $4(33 \%)$ \\
ASIA impairment scale & \\
A & $9(75 \%)$ \\
B & $3(25 \%)$ \\
Time since injury (years) & $22.3(15.7)$
\end{tabular}

ASIA American Spinal Injury Association 


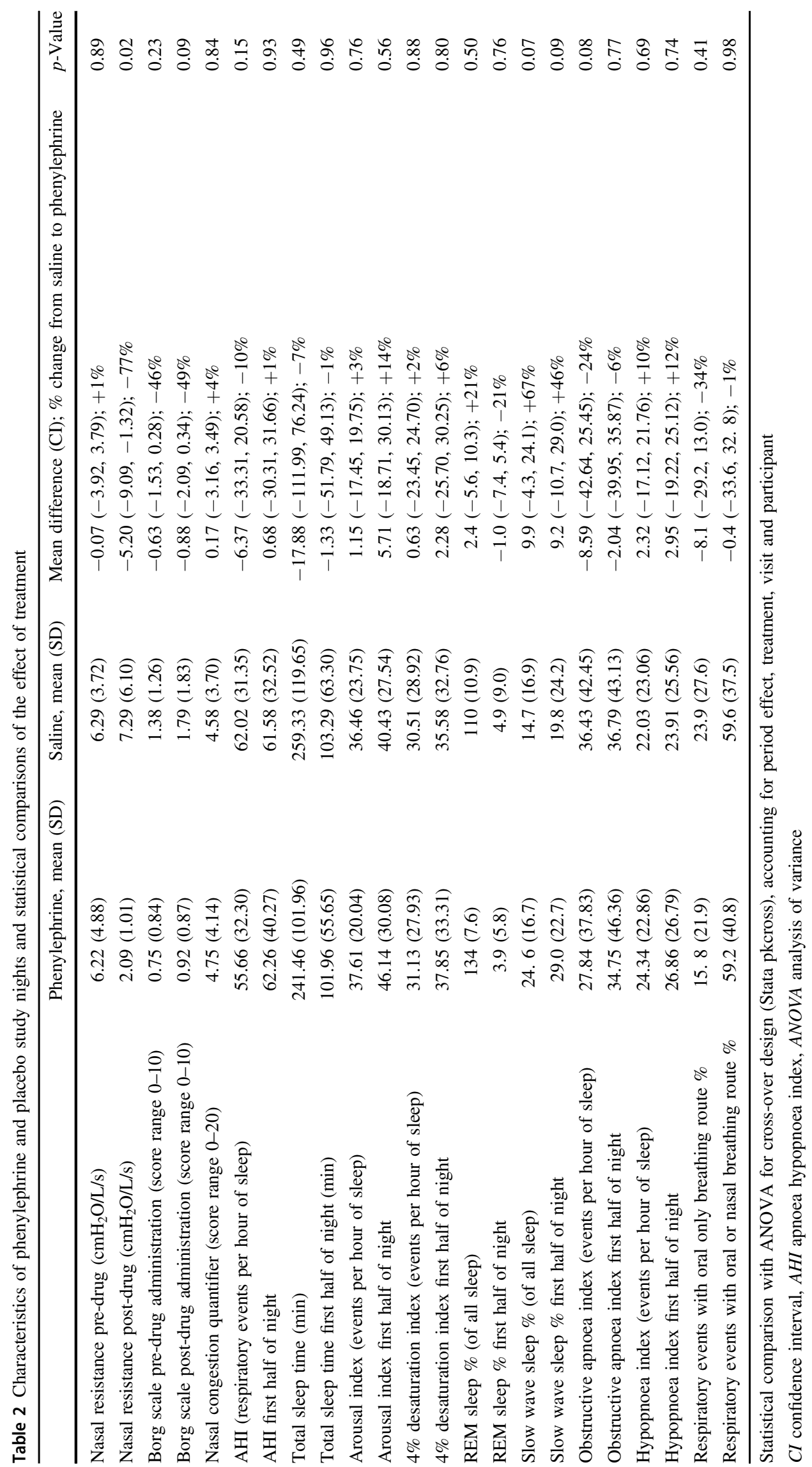




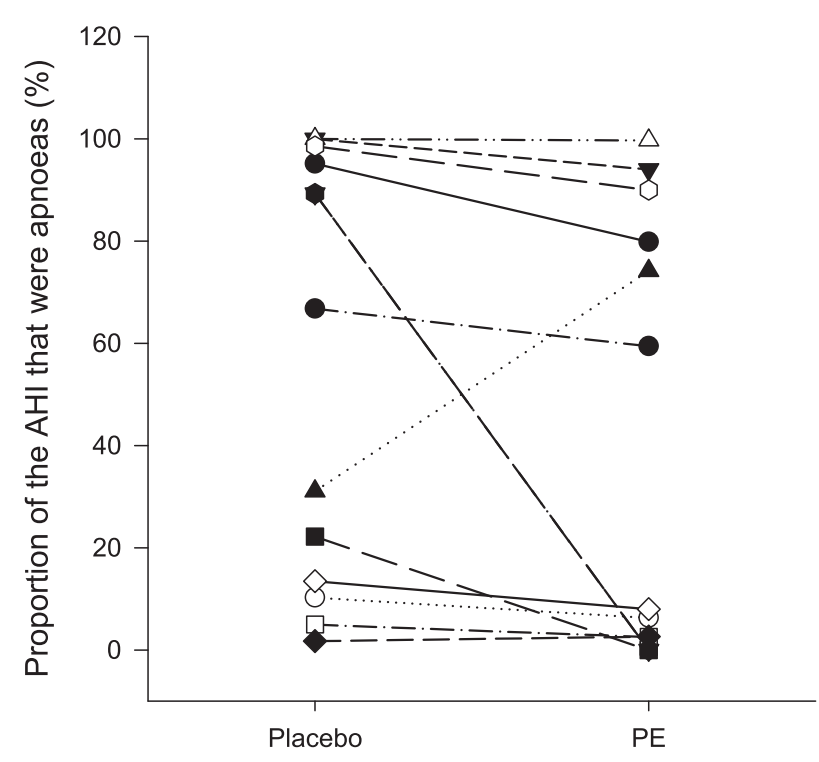

Fig. 2 The proportion of each participant's apnoea hypopnoea index that were scored as apnoeas

nasal blockage $(p=0.09 ;-0.88(-2.09,0.34)$ and the rate of obstructive apnoeas per hour $(p=0.15 ;-6.37(-33.31$, 20.58) were nonsignificantly reduced following PE while overnight slow wave sleep was nonsignificantly increased $(p=0.07 ; 9.88(-4.30,24.07)$ Table 2$)$. Sensitivity analyses confirmed no significant difference in AHI $(p=0.93)$ or total sleep time in the first half of the night $(p=0.96$ Table 2).

An exploratory review of the raw PSG data was undertaken, which demonstrated changes in sleep architecture and respiratory event severity with PE administration at an individual participant level. As illustrated in Fig. 2, nasal decongestion in all but one of the participants reduced respiratory event severity (apnoeas fell and hypopnoeas rose) defined as the proportion of the AHI that were apnoeas. The single participant whose percentage of apnoeas increased during the PE night had no REM sleep on the placebo night. Her/his AHI during REM was therefore 0 on placebo, 37.8 events/h on PE and her/his AHI during non-REM (NREM) was 41.2 on placebo falling to 31.7 after PE. The reduction in respiratory event severity with PE was not statistically significant $(p=0.28$; mean difference $-9.7 \%$ : $95 \%$ confidence interval $-28.5,9.1)$. If the participant without REM on the placebo night is removed, the mean reduction was larger at $14.5 \%$ but remained nonsignificantly different $(p=0.09$ : $-31.8,2.7)$.

\section{Discussion}

Administration of the decongestant PE was associated with significantly reduced nasal resistance, but no significant change in OSA. The reduction in nasal resistance confirms previous findings that elevated nasal resistance in people with tetraplegia is rapidly reduced following PE administration [16]. There was, however, no evidence that this acute physiological benefit reduced the AHI over either the first half or the whole night. PE may not be a suitable agent, or nasal resistance may not be a modifiable mechanism underpinning the increased prevalence of OSA in patients with SCI.

For the primary outcome measures of AHI and total sleep time measured in the first half of the night, there were no apparent differences in the group averages. While not statistically significant, over the whole night the AHI was six points lower with PE administration, and there was a tendency towards fewer obstructive apnoeas with PE (obstructive events were eight points lower overall following PE). As illustrated in Fig. 2, fewer obstructive events may indicate a reduction in event severity. OSA represents one end of a continuum from snoring to upper airway flow limitation to hyponoeas and apnoeas [23] and we speculate that the PE tended to reduce respiratory event severity. A tendency towards increased slow wave sleep is also consistent with an improvement in sleep quality.

Nasal congestion is a common and significant problem for patients with tetraplegia [24]. Although the reduction in nasal resistance did not result in significant changes in AHI, decreased congestion may have important implications for CPAP compliance, the gold standard treatment for OSA. Nasal congestion has been previously reported as contributing to CPAP intolerance [25] and investigating whether combining CPAP with an agent to reduce nasal resistance and subsequently increasing treatment adherence, should be investigated with future research.

In the able bodied, administering concurrent nasal decongestant and corticosteroid treatment reduced the AHI by $21 \%$ in a group of 21 people with OSA [17]. Treatment was administered twice daily for 1 week in people with normal nasal resistance in wakefulness. A switch from oral to nasal breathing route explained $64 \%$ of the reduction in AHI in their paper, but we did not observe any similar change (Table 2). Koutsourelakis et al. suggested that the medications prevented overnight increases in nasal resistance and that this drove the reduction in AHI [17]. These findings in the able-bodied differ to the current study findings in several ways. First, the severity of sleep apnoea was worse in our people with tetraplegia than the able bodied (AHIs of 56 and 31 events/h, respectively). Second, in the current study of people with tetraplegia and chronically elevated nasal resistance due to autonomic dysfunction, we aimed to 'reduce elevated resistance' as opposed to 'preventing nocturnal increases in nasal resistance' in the able bodied. It is possible that this was more difficult to achieve. Treatment in the able bodied also encompassed both 
decongestant and steroid treatment, over a 1-week period. The longer duration of treatment may have yielded greater benefit, however, this is likely only applicable to steroid treatment as the decongestant is short acting. The steroid effect may have been greater than that of the decongestant on AHI, or the combination may have been synergistic or potentiating. In the able bodied, steroid treatment alone has been shown to lower the AHI, reduce nasal congestion and improve day-time alertness [26]. Taken together, our current findings and the previous research suggest that reducing nasal congestion remains a worthwhile therapeutic target, but that PE is not the best single agent for reducing the AHI in people with tetraplegia and OSA. The emerging literature suggests that the pathophysiology of OSA in tetraplegia is different to what is observed in the non-disabled population [12-16]. Elevated nasal resistance remains an obvious therapeutic target and future research should consider testing steroid treatment and longer acting decongestant agents that do not elicit rebound or other substantive side effects. Similarly, nasal resistance reduction could be coupled with other interventions such as humidification [27] to facilitate better adherence with CPAP.

The current study is limited by its small sample size, but provides initial insights into the effects of PE in people with tetraplegia. An additional limitation of the current study may be the unknown duration and magnitude of reduced nasal resistance over time following administration of decongestant as this was only measured prior to sleep and assumptions made based on the reported drug half-life. Understanding the magnitude and time-course of any reduced nasal resistance during the sleep in this group of people with cervical SCI would permit more conclusive investigation in the relationship between airway resistance and AHI in people with in tetraplegia. Design and analysis of cross-over trials is not without challenge. The period effect and time trends should be accounted for with design and analysis. In this study, period was accounted for within the analysis (as was 'participant' for repeated measures), and adequate washout (short-acting treatment) and stability of the underlying condition (nasal resistance) were an integral element of the study design. There is debate in the statistical literature with respect to the best way to analyse the effects of cross-over trials, Stata offers one method using analysis of variance for $2 \times 2$ cross-over trials (pkcross), though other approaches include cluster level analyses, random effects and generalised estimating equations.

\section{Conclusions}

PE administration acutely reduced nasal resistance in people with tetraplegia and OSA but did not translate into a significant reduction in AHI during sleep. We would, however, argue that further research is warranted given the results of this preliminary study. Identification of an appropriate long-acting agent that reduced nasal congestion and was suitable for ongoing use, could reduce OSA severity as is observed with oral appliance use in the nondisabled.

\section{Data archiving}

Deidentified group data, as per informed consent, are all made available within the paper. Consent for sharing of individual participant data was not obtained.

Funding This research project was proudly supported by the Transport Accident Commission (Sleep Health in Quadriplegia Program) and received National Health and Medical Research Council (NHMRC) project grant funding (1065913).

Author contributions NSW was involved in the literature review, study design, data collection, analysis of data, manuscript preparation and review of the manuscript. DJE and ASJ were involved in study design, funding attainment, analysis of data and review of the manuscript. RS was involved in literature review, data collection, analysis of data, manuscript preparation and review of the manuscript. HM and $\mathrm{LB}$ were involved in data collection and review of the manuscript. CL and DB were involved in study design and review of the manuscript. MG was involved in study design, data collection, analysis of data and review of the manuscript. DJB was involved in literature review, study design, funding attainment, data collection, analysis of data, manuscript preparation and review of the manuscript.

\section{Compliance with ethical standards}

Conflict of interest DJE is supported by a NHMRC Senior Research Fellowship (1116942). ASJ is supported by an Australian Research Council Future Fellowship (FT100100203). DJE has a Commonwealth Government of Australia Cooperative Research Centre grant (industry partner: Oventus Medical), serves as a consultant for Bayer and has research support from Apnimed. The remaining authors declare that they have no conflict of interest.

Statement of ethics We certify that all applicable institutional and governmental regulations concerning the ethical use of human volunteers were followed during the course of this research.

Publisher's note: Springer Nature remains neutral with regard to jurisdictional claims in published maps and institutional affiliations.

\section{References}

1. AccessEconomics. The economic cost of spinal cord injury and traumatic brain injury in Australia. Report by Access Economics Pty Limited for the Victorian Neurotrauma Initiative, 2009.

2. Berlowitz DJ, Brown DJ, Campbell DA, Pierce RJ. A longitudinal evaluation of sleep and breathing in the first year after cervical spinal cord injury. Arch Phys Med Rehabil. 2005;86:1193-9. 
3. Giannoccaro MP, Moghadam KK, Pizza F, Boriani S, Maraldi NM, Avoni P, et al. Sleep disorders in patients with spinal cord injury. Sleep Med Rev. 2013;17:399-409.

4. Berlowitz DJ, Spong J, Gordon I, Howard ME, Brown DJ. Relationships between objective sleep indices and symptoms in a community sample of people with tetraplegia. Arch Phys Med Rehabil. 2012;93:1246-52.

5. Biering-Sorensen F, Biering-Sorensen M. Sleep disturbances in the spinal cord injured: an epidemiological questionnaire investigation, including a normal population. Spinal Cord. 2001;39:505-13.

6. Spong J, Graco M, Brown DJ, Schembri R, Berlowitz DJ. Subjective sleep disturbances and quality of life in chronic tetraplegia. Spinal Cord. 2015;53:636-40.

7. Sajkov D, Marshall R, Walker P, Mykytyn I, McEvoy RD, Wale J, et al. Sleep apnoea related hypoxia is associated with cognitive disturbances in patients with tetraplegia. Spinal Cord. 1998;36:231-9.

8. Schembri R, Spong J, Graco M, Berlowitz DJ, team Cs. Neuropsychological function in patients with acute tetraplegia and sleep disordered breathing. Sleep 2017;40:zsw037.

9. Eckert DJ, White DP, Jordan AS, Malhotra A, Wellman A. Defining phenotypic causes of obstructive sleep apnea. Identification of novel therapeutic targets. Am J Respir Crit Care Med. 2013;188:996-1004.

10. Fuller DD, Lee KZ, Tester NJ. The impact of spinal cord injury on breathing during sleep. Respir Physiol Neurobiol. 2013;188:344-54.

11. Fang X, Goh MY, O'Callaghan C, Berlowitz D. Relationship between autonomic cardiovascular control and obstructive sleep apnoea in persons with spinal cord injury: a retrospective study. Spinal Cord Ser Cases. 2018;4:29.

12. Wijesuriya NS, Gainche L, Jordan AS, Berlowitz DJ, LeGuen M, Rochford PD, et al. Genioglossus reflex responses to negative upper airway pressure are altered in people with tetraplegia and obstructive sleep apnoea. J Physiol. 2018;596:2853-64.

13. Sankari A, Vaughan S, Bascom A, Martin JL, Badr MS. Sleep disordered breathing and spinal cord injury- a state of the art review. Chest 2018;155:438-45.

14. O'Donoghue FJ, Meaklim H, Bilston L, Hatt A, Connelly A, Jackson G, et al. Magnetic resonance imaging of the upper airway in patients with quadriplegia and obstructive sleep apnea. J Sleep Res. 2018;27:e12616.

15. Wijesuriya NS, Lewis C, Butler JE, Lee BB, Jordan AS, Berlowitz DJ, et al. High nasal resistance is stable over time but poorly perceived in people with tetraplegia and obstructive sleep apnoea. Respir Physiol Neurobiol. 2017;235:27-33.
16. Gainche L, Berlowitz DJ, LeGuen M, Ruehland WR, O'Donoghue FJ, Trinder J, et al. Nasal resistance is elevated in people with tetraplegia and is reduced by topical sympathomimetic administration. J Clin Sleep Med. 2016;12:1487-92.

17. Koutsourelakis I, Minaritzoglou A, Zakynthinos G, Vagiakis E, Zakynthinos S. The effect of nasal tramazoline with dexamethasone in obstructive sleep apnoea patients. Eur Respir J. 2013;42:1055-63.

18. Verse T, Pirsig W. Impact of impaired nasal breathing on sleepdisordered breathing. Sleep Breath. 2003;7:63-76.

19. Burns SP, Little JW, Hussey JD, Lyman P, Lakshminarayanan S. Sleep apnea syndrome in chronic spinal cord injury: associated factors and treatment. Arch Phys Med Rehabil. 2000;81:1334-9.

20. Kirshblum SC, Burns SP, Biering-Sorensen F, Donovan W, Graves DE, Jha A, et al. International standards for neurological classification of spinal cord injury (revised 2011). J Spinal Cord Med. 2011;34:535-46.

21. Berry RB, Budhiraja R, Gottlieb DJ, Gozal D, Iber C, Kapur VK, et al. Rules for scoring respiratory events in sleep: update of the 2007 AASM Manual for the Scoring of Sleep and Associated Events. Deliberations of the Sleep Apnea Definitions Task Force of the American Academy of Sleep Medicine. J Clin Sleep Med. 2012;8:597-619.

22. Stull DE, Meltzer EO, Krouse JH, Roberts L, Kim S, Frank L, et al. The congestion quantifier five-item test for nasal congestion: refinement of the congestion quantifier seven-item test. Am J Rhinol Allergy. 2010;24:34-8.

23. Jordan AS, McSharry DG, Malhotra A. Adult obstructive sleep apnoea. Lancet. 2014;383:736-47.

24. Lung MA, Wang JC. Autonomic nervous control of nasal vasculature and airflow resistance in the anaesthetized dog. J Physiol. 1989;419:121-39.

25. Berlowitz DJ, Spong J, Pierce RJ, Ross J, Barnes M, Brown DJ. The feasibility of using auto-titrating continuous positive airway pressure to treat obstructive sleep apnoea after acute tetraplegia. Spinal Cord. 2009;47:868-73.

26. Kiely JL, Nolan P, McNicholas WT. Intranasal corticosteroid therapy for obstructive sleep apnoea in patients with co-existing rhinitis. Thorax. 2004;59:50-5.

27. Koutsourelakis I, Vagiakis E, Perraki E, Karatza M, Magkou C, Kopaka M, et al. Nasal inflammation in sleep apnoea patients using CPAP and effect of heated humidification. Eur Respir J. 2011;37:587-94. 